\title{
Molecular effects of Lapatinib in the treatment of HER2 overexpressing oesophago-gastric adenocarcinoma
}

\begin{abstract}
Nadeera De Silva ${ }^{1}$, Laura Schulz ${ }^{1}$, Anna Paterson ${ }^{1}$, Wendi Qain $^{2}$, Maria Secrier ${ }^{3}$, Edmund Godfrey ${ }^{4}$, Heok Cheow ${ }^{4}$, Maria O'Donovan ${ }^{5}$, Pierre Lao-Sirieix ${ }^{1}$, Minesh Jobanputra ${ }^{6}$, Daniel Hochhauser ${ }^{7}$, Rebecca Fitzgerald ${ }^{\star}, 1$ and Hugo Ford ${ }^{8}$

${ }^{1}$ MRC Cancer Unit, Hutchison/MRC Research Centre, University of Cambridge, Hills Road, Cambridge CB2 OXZ, UK; ${ }^{2}$ Cambridge Clinical Trials Unit, Coton House, Cambridge University Hospitals NHS Foundation Trust, Hills Road, Cambridge CB2 0QQ, UK; ${ }^{3}$ Cancer Research UK Cambridge Institute, University of Cambridge, Li Ka Shing Centre, Robinson Way, Cambridge CB2 ORE, UK; ${ }^{4}$ Department of Radiology, Cambridge University Hospitals NHS Foundation Trust, Cambridge Biomedical Campus, Hills Road, Cambridge CB2 0QQ, UK; ${ }^{5}$ Department of Pathology, Cambridge University Hospitals NHS Foundation Trust, Cambridge Biomedical Campus, Hills Road, Cambridge CB2 0QQ, UK; ${ }^{6}$ Glaxosmithkline, Stevenage SG1 2NY, UK; ${ }^{7}$ University College Hospital, 235 Euston Road, London NW1 2BU, UK and ${ }^{8}$ Department of Oncology, Cambridge University Hospitals NHS Foundation Trust, Cambridge Biomedical Campus, Hills Road, Cambridge CB2 0QQ, UK
\end{abstract}

Background: Lapatinib, a dual EGFR and HER2 inhibitor has shown disappointing results in clinical trials of metastatic oesophagogastric adenocarcinomas (OGAs), and in vitro studies suggest that MET, IGFR, and HER3 confer resistance. This trial applied Lapatinib in the curative neoadjuvant setting and investigated the feasibility and utility of additional endoscopy and biopsy for assessment of resistance mechanisms ex vivo and in vivo.

Methods: Patients with HER2 overexpressing OGA were treated for 10 days with Lapatinib monotherapy, and then in combination with three cycles of Oxaliplatin and Capecitabine before surgery. Endoscopic samples were taken for molecular analysis at: baseline including for ex vivo culture + / - Lapatinib to predict in vivo response, post-Lapatinib monotherapy and at surgery. Immunohistochemistry (IHC) and proteomic analysis was performed to assess cell kinetics and signalling activity.

Results: The trial closed early $(n=10)$ due to an anastomotic leak in two patients for which a causative effect of Lapatinib could not be excluded. The reduction in Phosphorylated-HER2 (P-HER2) and P-EGFR in the ex vivo-treated biopsy demonstrated good correlation with the in vivo response at day 10 . Proteomic analysis pre and post-Lapatinib demonstrated target inhibition (P-ERBB2, P-EGFR, P-PI3K, P-AKT, and P-ERK) that persisted until surgery. There was also significant correlation between the activation of MET with the level of P-Erk $(P=0.0005)$ and P-PI3K:T-PI3K (total PI3K) ratio $(P=0.0037)$. There was no significant correlation between the activation status of IGFR and HER3 with downstream signalling molecules.

Conclusions: Additional endoscopy and biopsy sampling for multiple biomarker endpoints was feasible and confirmed in vitro data that MET is likely to be a significant mechanism of Lapatinib resistance in vivo.

The incidence of oesophago-gastric adenocarcinoma (OGA), has been increasing rapidly (CancerResearchUK, 2015), but 5 year overall survival remains poor, and it is the sixth most common cause of cancer related death. Locally advanced (non-metastatic) tumours are treated by surgery, and the addition of chemotherapy has improved 5 year overall survival from 23 to $36 \%$ (Cunningham

*Correspondence: Professor R Fitzgerald; E-mail: rcf29@cam.ac.uk

Received 29 May 2015; revised 20 August 2015; accepted 27 August 2015; published online 20 October 2015

(c) 2015 Cancer Research UK. All rights reserved 0007-0920/15 
et al, 2008). Oesophago-gastric adenocarcinoma (16.6\%) overexpress HER2 (Van Cutsem et al, 2014) and data suggests that this upregulation occurs early in the process of carcinogenesis at the stage of pre-invasive Barrett's oesophagus and gastric intraepithelial neoplasia (Fassan et al, 2012; Paterson et al, 2013a). Trastuzumab, a monoclonal antibody to HER2, has shown significant activity in HER2 overexpressing gastric cancer, although the clinical use so far has been restricted to the metastatic setting (Bang et al, 2010). Lapatinib, a dual EGFR and HER2 receptor tyrosine kinase inhibitor (TKIs) has also been investigated in metastatic disease but response activity does not appear to be as great as for Trastuzumab. A Phase II monotherapy study showed a very modest response rate to single agent Lapatinib (Iqbal et al, 2011), and when used in combination with chemotherapy (paclitaxel) in the TyTan trial, only modest gains in survival were observed (11 m vs $8.9 \mathrm{~m} P=0.1044$; Satoh et al, 2014). The TRIO013/LOGiC trial, a randomised Phase III trial evaluating the combination of Capecitabine/Oxaliplatin with or without Lapatinib in the first-line treatment of metastatic OGAs demonstrated a nonsignificant increase in overall survival in the Lapatinib arm (overall survival of $12.2 \mathrm{~m}$ vs $10.5 \mathrm{~m}, P=0.35$; Hecht et al, 2013) whilst the recent Phase II trial comparing Lapatinib with or without Capecitabine in the second line setting demonstrated a very modest response rates of $11 \%$ in combination with chemotherapy and no responders in the Lapatinib monotherapy arm (Lorenzen et al, 2015). These results point to significant primary resistance mechanisms.

Targetting HER2 in gastric cancer cell lines and xenografts with TKIs or monoclonal antibodies results in inhibition of the phosphorylated receptor and downstream signalling through proliferative pathways such as PI3K (Wainberg et al, 2010; Chen et al, 2012). However, signalling through alternative receptor tyrosine kinases such as MET (Hepatocyte Growth Factor Receptor), HER3, and IGF1R (Insulin-like Growth Facotor Receptor-1) were noted to produce resistance to Lapatinib (Zhang et al, 2014). Inhibiting MET or IGFR activation with their corresponding TKI restored Lapatinib sensitivity, which was associated with a decrease in downstream activation of Akt and Erk in vitro. Similar work on cell lines demonstrated that the use of TKI combinations based on RTK activation status resulted in greater inhibition of proliferation of OGA cell lines (Paterson et al, 2013b). Though these in vitro studies shed some insight into the mechanisms of Lapatinib resistance, they may not represent the situation in vivo. Studies have explored the use of patient derived xenografts and organoids for drug sensitivity testing (Dobbin et al, 2014; Sachs and Clevers, 2014), but the major disadvantage with these methods is the time required to perform these assays, which means that that results cannot be delivered in a timely manner to inform patient management. Simple and rapid methods need to be developed to provide biomarker evidence to help guide clinical decision making. Options include the use of ex vivo-treated tumour biopsies, or repeat biopsies after window periods of therapy. This is particularly feasible for OGA that is accessible endoscopically. The Lapatinib in early oesophago-gastric cancer trial assessed Lapatinib as first-line therapy in the curative setting for OGA and aimed to:

1. To assess the safety and toxicity profile of Lapatinib with chemotherapy, when used peri-operatively for patients on a radical pathway.

2. To test whether an ex vivo assay examining the ability of Lapatinib to suppress HER2 and EGFR could predict in vivo response to 10 days of Lapatinib monotherapy.

3. To establish if assays of downstream signalling performed on biopsies taken before and after 10 days of Lapatinib monotherapy could indicate mechanisms of resistance/sensitivity to the drug

4. Correlate the molecular indicators of response to imaging criteria (functional ${ }^{18}$ FDG-PET and CT imaging) along with clinical outcome measures such as R0 resection rate, complete pathological response and overall survival.

\section{MATERIALS AND METHODS}

Trial design. This was a two-centre open label trial of neoadjuvant treatment with Lapatinib alone and then in combination with Oxaliplatin and Capecitabine in HER2 expressing oesophageal or gastric cancers undergoing curative therapy.

All patients provided written informed consent before entry on the trial, and the study protocol was approved by a multi-centre research ethics committee, and a clinical trial authorisation was granted (number 12854/0235/001-0001) with Lapatinib, Capecitabine, and Oxaliplatin listed as Investigational Medicinal Products.

Power calculation. This is a molecular biomarker study and thus designed to examine molecular rather than clinical endpoints. The primary objective was to compare the molecular response in biopsies taken pre-treatment, which were then treated with Lapatinib ex vivo with the molecular response in a biopsy taken after 10 days of oral Lapatinib. It was anticipated that concordant response outcomes (no change or decrease) would be observed for at least 80 to $85 \%$ patients, with a $5 \%$ significance (one-sided) level and $80 \%$ power, 13 patients would need to be recruited.

Patient selection. Patients were enroled with histologically confirmed OGA that over expressed HER2, defined as either $3+$ staining intensity for HER2 on immunohistochemistry (IHC), or $2+$ staining but shown to have HER2 amplification by FISH (Bang et al, 2010). All patients were deemed to have surgically resectable disease on presentation based on CT, PET CT, endoscopic ultrasound (oesophageal cases), and staging laparoscopy (gastric cases), and needed to be fit for both chemotherapy and surgery, with adequate haematological parameters $\left(\mathrm{WBC} \geqslant 3.0 \times 10^{9} 1^{-1}\right.$, Plts $\left.\geqslant 100 \times 10^{9} 1^{-1}, \mathrm{Hb} \geqslant 9 \mathrm{~g} \mathrm{dl}^{-1}\right)$, renal function (measured or calculated Cr clearance $\geqslant 60 \mathrm{ml} \mathrm{min}^{-1}$ ) and liver function (Bilirubin $\leqslant 1.5 \times \mathrm{ULN}$, ALT/AST $\leqslant 1.5 \times \mathrm{ULN}$. $\mathrm{ALP} \leqslant 2.5 \times \mathrm{ULN})$. They needed to be able to swallow oral medication and be over 18 years of age. Patients with abnormal cardiac function determined by echocardiography/MUGA scan, or a history of interstitial lung disease were excluded. Those who had previously received chemotherapy or Lapatinib were excluded as were those with a known G6PD deficiency. Cases of known HIV, Hepatitis C or B infection were also excluded.

Treatment. Patients initially received 10 days of treatment with Lapitinib $1250 \mathrm{mg}$ once a day. This period was used to assess the effect of Lapatinib alone on the tumour (Figure 1). From day 11, patients went on to receive three cycles of Oxaliplatin

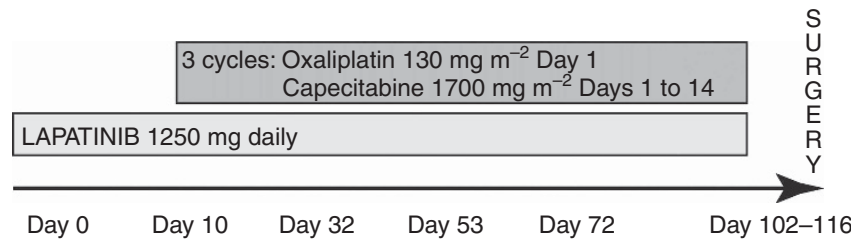

\section{PET CT PET CT}

BIOPSY BIOPSY

BIOPSY

CT

CT

Figure 1. Study design where patients undergo a baseline biopsy and ex vivo sample was taken at D0. Patients then received 10 days of Lapatinib monotherapy after which a repeat biopsy and functional imaging was performed. Patients went on to receive three cycles of Oxaliplatin and Capecitabine concurrent with Lapatinib on a 21-day cycle, followed by definitive surgery. 
$\left(130 \mathrm{mg} \mathrm{m}^{-2}\right)$ and Capecitabine $1700 \mathrm{mg} \mathrm{m}^{-2}$ whilst continuing Lapatinib. On completion of neoadjuvant therapy, the patient went on to have definitive surgery in the form of an oesophagectomy or gastrectomy depending on the location of the tumour.

Assessment. Toxicity was assessed using the National Cancer Institute Common Terminology Criteria for Adverse Events Version 3.0. Survival was determined by either confirmed date of death, or censored at the last recorded trial follow-up event. The Kaplan-Meier method was adopted for the survival analysis.

The PET CT scans were performed on day D0 and day D10 after treatment with Lapatinib monotherapy. The fall in the absolute maximum standard uptake value and for a region of interest of $1.5 \mathrm{~cm}$ diameter circle at D0 and D10 was reported by an Administration of Radioactive Substances Committee (ARSAC) accredited radiologist. A PET response was defined as a reduction of $>35 \%$ in the standard uptake value (Lordick et al, 2007). The CT scans were performed on patients on D0 and on D72. Tumour response to therapy was evaluated between the two time points using RESCIST 1.1.

Biopsy analysis. Biopsies at baseline, D10 and surgery were taken and the specimen was split equally into two parts. One part was fresh frozen and stored in readiness for processing using the Collaborative Enzyme Enhanced Reactive Immunoassay (CEER; Prometheus Laboratories Inc, San Diego, USA) and the other part of the biopsy was formalin fixed for H\&E and IHC analyses.

At baseline, a further tissue sample was treated with Lapatinib ex vivo. This was done by placing the biopsy tissue measuring $\sim 0.2 \mathrm{~cm}$, on to a mesh placed in a well which was then filled with Dulbecco's Modified Eagles' Medium (Gibco, Invitrogen, Paisley, UK) medium and 10 um Lapatinib (GlaxoSmithKline, Uxbridge, UK) or with a control DMSO (Fisher Scientific, Loughborough, UK) so as to provide an air-liquid interface. This was then incubated at $37^{\circ} \mathrm{C}$ for $1 \mathrm{~h}$, and then fixed in $10 \%$ neutral buffered formalin pots, to create paraffin blocks ready for IHC.

All samples were stained by IHC for P-HER2, HER2, P-EGFR, P-Erk, P-Akt, (Cell Signalling, Danvers, MA, USA), EGFR (Novacastra), and MET (Santa Cruz Biotechnology) using the Leica Bond Max Automated Immunohistochemistry System (Wetzlar, Germany). Staining was scored by two independent reviewers using intensity $(0=$ no reactivity or membranous staining of tumour cell, $1=$ faint/barely perceptible membranous staining of $\geqslant 10 \%$ of tumour cells, $2=$ weak to moderate complete, basolateral or lateral membranous reactivity in $\geqslant 10 \%$ of tumour cells, $3=$ strong complete, basolateral or lateral membranous reactivity in $\geqslant 10 \%$ of tumour cells; Koopman et al, 2015). Scores of 2 and 3 were considered positive whereas 0 and 1 were negative, and a response was defined as a positive score becoming negative (Zhang et al, 2014). A response was defined as a change from a positive IHC score $(2+$ or $3+)$ to a negative score $(0+$ or $1+)$ for P-HER2 and P-EGFR. Proliferation was assessed by staining for Ki67 (Leica, Wetzlar Germany), and apoptosis assessed by staining for cleaved caspase 3 (Cell Sginalling, Danvers, MA, USA) and by using the TUNEL assay (Millipore, Consett UK). A score of 1-5 was given based on the percentage of tumour cells staining for Ki67 $(1=0-20 \%, 2=21-40 \%, 3=41-60 \%, 4=61-80 \%, 5=>81 \%)$. Scoring cleaved caspase 3 and the TUNEL assay was done my counting all positively staining cancer cells in 5 high-power $(40 \times)$ fields of view by 2 independent scorers, and the average of all 10 scores was taken for the purposes of analysis ( $\mathrm{Li}$ et al, 2008).

All frozen samples were assayed using the CEER platform (Prometheus Laboratories, San Diego, USA) for receptor tyrosine kinase activation (EGFR, HER2, p95, HER3, IGFR, and MET) and downstream signalling. This could not be done with the ex vivo biopsy as it was formalin fixed and further material was not available.
Genomic analysis. Whole genome sequencing was available for one patient's treatment naive tumour. DNA was extracted from fresh frozen tissue samples using the Qiagen DNA Mini Kit (Qiagen) as per protocol and sequenced on the Illumina HiSeq platform. Paired DNA from blood was sequenced to allow somatic variants to be distinguished from those in the germline. For alignment against GRCh37 reference from Ensembl v71 BWA was used. Variant calling was performed with Somatic Sniper (V1.0.2) and a variety of filters was used to exclude low quality calls (Larson et al, 2012; Weaver et al, 2014). The single nucleotide variation data was assessed using DAVID Bioinformatics resource Version 6.7 (National Institute of Allergy and Infectious Diseases). Functional annotation was assessed using the Gene Ontology tools and KEGG pathway analysis. ASCAT-NGS v.2.1 (Van Loo et al, 2010; Nik-Zainal et al, 2012) was used to infer tumour copy number data, and a combination of both copy number and single nucleotide variation data was analysed in cloneHD to assess clonality (Fischer et al, 2014).

Statistical analysis. For the assessment of the ex vivo assay by IHC, a molecular response was defined as a positive P-HER2 or P-EGFR $(2+$ or $3+)$ becoming a negative score $(0+$ or $1+$; Zhang et al, 2014). A Kappa correlation coefficient was calculated to assess agreement between ex vivo and D10 in vivo response, and between the two reviewers for these scores. Wilcoxon-Rank Sums analysis was performed, between baseline tumour IHC characteristics (HER2, EGFR, and MET) and predefined clinical outcome (RECIST response, complete pathological response, R0 resection).

HER2, p95, and EGFR activation (phosphorylated to total protein ratio) using CEER data was assessed at baseline, D10 and surgery and the Wilcoxon-Signed Rank test was used to assess changes between baseline and D10 as well as baseline and surgery.

Correlation between MET, IGFR, and HER3 activation (phosphorylated to total protein ratio) was correlated with the PI3K, P-Erk, and P-Akt activation (absolute levels of P-Erk and P-Akt was used as the total protein level was not available for these two molecules) using the Spearman Rank Correlation.

\section{RESULTS}

A total of 10 patients were recruited to the trial. The median age of the 10 patients was 61.7 years, with a preponderance for males (70\%) which is in keeping with the epidemiology of the disease. Eight of the patients entering the trial had a performance status of 0 , and the remaining two a performance status of 1 . The primary site of disease was the gastro-oesophageal junction in six of the cases and the majority of these tumours were poorly differentiated (Table 1). All patients bar one had a HER2 IHC score of $3+$ and only one patient had a tumour with an IHC score of 2, which was subsequently confirmed to be amplified by FISH.

The 10-day Lapatinib monotherapy window phase was well tolerated with $100 \%$ of patients receiving the intended Lapatinib dose with no $\geqslant$ grade 3 toxicity. With regards to the neoadjuvant period of chemotherapy with concurrent Lapatinib, $80 \%$ of patients received all three cycles of chemotherapy. These toxicities are summarised in Table 2 .

The most common adverse effects noted were nausea and diarrhoea. These are in keeping with the known individual toxicities of the drugs, especially Capecitabine and Lapatinib. Three patients experienced grade 4 toxicity. This included two patients who experienced anastomotic leaks. One of these patients died after developing a chest infection. This leak rate of $20 \%$ was higher than expected given that both tertiary centres had leak rates in keeping with the national oesophago-gastric audit of 5\%, and as a result the independent data monitoring committee agreed to suspend recruitment to the study after 10 patients had been 
Table 1. Demographic data on patient cohort, with primary site, HER2 scoring, tumour differentiation and stage

\begin{tabular}{|c|c|}
\hline \multicolumn{2}{|c|}{$n=10$} \\
\hline \multicolumn{2}{|l|}{ Age (years) } \\
\hline $\begin{array}{l}\text { Mean } \\
\text { s.d. } \\
\text { Range }\end{array}$ & $\begin{array}{c}61.7 \\
9.4 \\
49.7-75.2\end{array}$ \\
\hline \multicolumn{2}{|l|}{ Sex } \\
\hline $\begin{array}{l}\text { Male } \\
\text { Female }\end{array}$ & $\begin{array}{l}7 \\
3\end{array}$ \\
\hline \multicolumn{2}{|l|}{ ECOG performance status } \\
\hline $\begin{array}{l}0 \\
1\end{array}$ & $\begin{array}{l}8 \\
2\end{array}$ \\
\hline \multicolumn{2}{|l|}{ Site of primary } \\
\hline $\begin{array}{l}\text { Oesophagus } \\
\text { Stomach } \\
\text { GOJ type } 1 \\
\text { GOJ type } 2 \\
\text { GOJ type } 3\end{array}$ & $\begin{array}{l}3 \\
1 \\
1 \\
3 \\
2\end{array}$ \\
\hline \multicolumn{2}{|l|}{ HER2 staining } \\
\hline $\begin{array}{l}3+ \\
2+\text { with FISH amplification }\end{array}$ & $\begin{array}{l}9 \\
1 \\
\end{array}$ \\
\hline \multicolumn{2}{|l|}{ Tumour differentiation } \\
\hline $\begin{array}{l}\text { Moderate } \\
\text { Poor }\end{array}$ & $\begin{array}{l}4 \\
6\end{array}$ \\
\hline \multicolumn{2}{|l|}{ T stage } \\
\hline $\begin{array}{l}\text { T2 } \\
\text { T3 }\end{array}$ & $\begin{array}{l}1 \\
7\end{array}$ \\
\hline \multicolumn{2}{|l|}{$\mathrm{N}$ stage } \\
\hline $\begin{array}{l}\text { N0 } \\
\text { N1 } \\
\text { N2 }\end{array}$ & $\begin{array}{l}7 \\
2 \\
1\end{array}$ \\
\hline \multicolumn{2}{|l|}{ M stage } \\
\hline MO & 0 \\
\hline
\end{tabular}

enroled (Chadwick et al, 2013). Median overall survival was 32.4 months, and median progression free survival was 16.4 months. There were no responses by PET at D10 in the maximal standard uptake value of for the maximal region of interest $\left(1.5 \mathrm{~cm}^{2}\right.$ circle). Fifty per cent of patients had a partial response on CT after neoadjuvant therapy, and the rest had stable disease.

\section{Molecular analysis}

Immunohistochemistry. Tumour specimens were stained for HER2, EGFR, P-HER2, P-EGFR, and MET, as well as downstream targets including P-Erk, P-Akt and examples of staining patterns are demonstrated in Figure 2.

Assessment of the ex vivo assay. The ex vivo assay was used to determine whether it is possible to predict in vivo response after 10 days of Lapatinib monotherapy.

There was good agreement between the two independent scorers. Three samples were inadequate specimens and so paired data was available for nine cases for P-HER2 and eight for P-EGFR. The ex vivo and D10 molecular response correlated $89 \%$ of the time for P-HER2 and $100 \%$ for P-EGFR that meets the predefined aim of $75 \%$ (Table 3 ).

Comparison of molecular and clinical outcomes. As an alternative to the ex vivo culture assay, the IHC results on biopsies sampled over time were correlated to clinical outcome measures to
Table 2. Adverse events of $\geqslant$ grade 3 experienced by patients during their monotherapy window, and concurrent chemotherapy treatment

\begin{tabular}{|l|l|}
\hline \multicolumn{2}{|c|}{$n=10$} \\
\hline Lapatinib monotherapy \\
\hline Lapatinib monotherapy toxicities \\
\hline Lapatinib and chemotherapy \\
\hline Anastomotic leak & 2 \\
Fainting & 1 \\
Mucositis & 1 \\
Candida infection & 1 \\
Nausea & 2 \\
Atelectasis & 1 \\
Dyspepsia & 1 \\
Diarrhoea & 2 \\
Gastrointestinal Pain & 1 \\
Small bowel obstruction & 1 \\
Weight loss & 1 \\
Chest infection & 1 \\
Hypoxia & 1 \\
Neutropenia & 1 \\
\hline
\end{tabular}

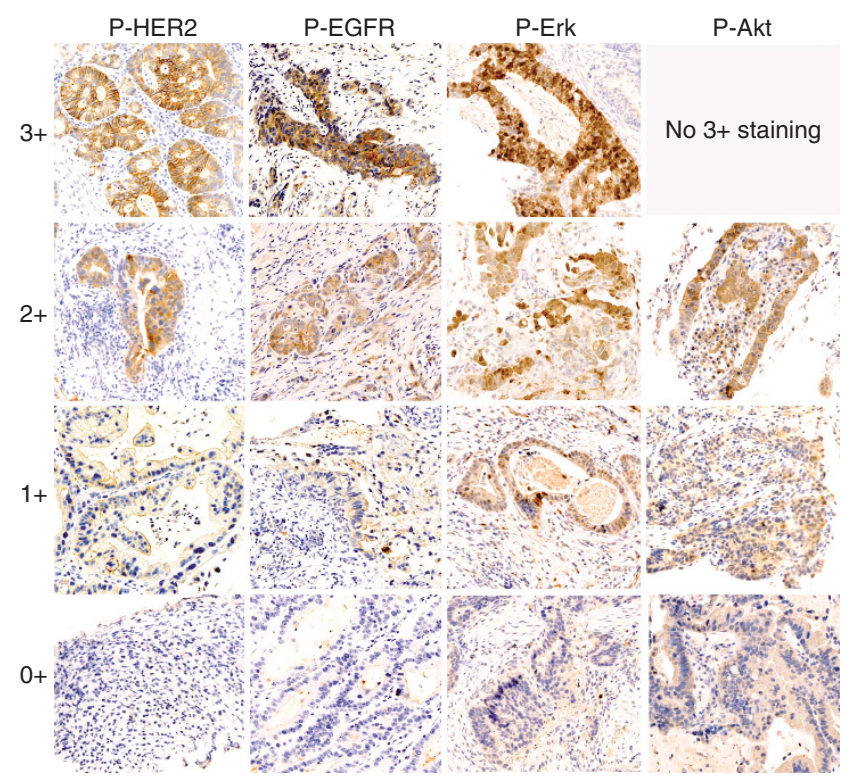

Figure 2. Immunohistochemistry for the phosphorylated species for the RTKs P-HER2, P-EGFR and the downstream molecules P-Erk and P-Akt. Examples of staining performed using the Axio Slide Scanner (Oberkochen, Germany) at $40 \times$ and images at a $35 \%$ digital zoom. No $3+$ staining was observed for P-Akt.

assess if any of these assays could be a potential biomarker for response to Lapatinib therapy.

The baseline score for HER2, EGFR, and MET (potential resistance mechanism) was related to clinical outcome measures. Our hypothesis was that stronger HER2 and EGFR staining tumours would be more responsive to Lapatinib, whereas tumours with strong MET expression would be resistant to therapy. Wilcoxon-Rank Sum Analysis failed to show any association between baseline HER2 scores and the clinical outcomes by RECIST response, R0 resection rate, or complete pathological response $(P=1.000, P=0.660$, and $P=0.606$, respectively). Nor was there any associations with baseline EGFR score $(P=0.535$, $P=0.087$, and $P=0.702)$ or MET scores $(P=0.399, P=0.615$, 0.615 , respectively). On-target response did not correlate with progression free survival or overall survival based on an IHC 
response (HER2-based response OS $P=0.2278$ PFS $P=0.6650$; EGFR-based response OS $P=0.3938$, PFS $P=0.3173$ ) or CEER response (HER2-based response OS $P=0.4126$, PFS $P=0.2216$; EGFR-based response OS $P=0.2922$, PFS $P=0.1486$ ).

CEER proteomic analysis. The target effect of Lapatinib was examined at baseline, D10, and surgery using the CEER assay (Figure 3). There was a significant drop in the P-HER2:T-HER2, and P-EGFR:T-EGFR ratios after only 10 days of Lapatinib monotherapy $(P=0.0039$ and $P=0.0195$, respectively). All 10 patients demonstrated reductions in HER2 activation by day 10 and 9 of 10 patients had a reduction in EGFR activation. This reduction

Table 3. Comparison of the ex vivo response to Lapatinib fo P-HER2 and P-EGFR, with that seen after 10 days of in vivo Lapatinib monotherapy

\begin{tabular}{|c|c|c|c|}
\hline & & \multicolumn{2}{|c|}{ Ex vivo } \\
\hline \multicolumn{2}{|l|}{ P-HER2 } & No change & Response \\
\hline \multirow[t]{3}{*}{ In vivo } & No change & 6 & 0 \\
\hline & Response & 1 & 2 \\
\hline & & \multicolumn{2}{|c|}{ Ex vivo } \\
\hline \multicolumn{2}{|l|}{ P-EGFR } & No change & Response \\
\hline \multirow[t]{2}{*}{ In vivo } & No Change & 7 & 0 \\
\hline & Response & 0 & 1 \\
\hline \multicolumn{4}{|c|}{$\begin{array}{l}\text { Abbreviation: } \mathrm{IHC}=\text { immunohistochemistry. Kappa score for P-HER2: between scorers } \\
\text { (weighted) }=0.5902 \text {; between ex vivo and } \mathrm{D} 10 \mathrm{HHC} \text { score }=0.7273 \text {; correlation between } \\
\text { ex vivo and } \mathrm{D} 10 \text { in vivo }=88.9 \% \text {. Kappa Score for P-EGFR: between scorers }=0.832 \text {; between } \\
\text { ex vivo and D10 IHC score }=1 \text {; correlation between ex vivo and D10 in vivo }=100.0 \% \text {. }\end{array}$} \\
\hline
\end{tabular}

in growth factor signalling persisted to the time of surgery and EGFR signalling remained significantly inhibited $(P=0.0391)$, despite a 30 day wash out period between completing neoadjuvant therapy and surgery. HER2 signalling (PI3K, P-AKT, and P-ERK) continued to be lower at the time of surgery compared with baseline, although the difference did not reach significance $(P=0.4961)$. The truncated form of HER2, p95HER2 lacks the extracellular domain, and significant inhibition was again noted at D10, which persisted through to surgery ( $P=0.0078$ and $P=0.0234$, respectively).

There was a reduction in P-PI3K:T-PI3K activation that mirrors the response in HER2 and EGFR, with significant dampening in signalling between baseline and both D10 as well as surgery $(P=0.03$ and $P=0.01$, respectively). Only phosphorylated assays were available for Akt and Erk, and though there was a reduction in median levels, this did not reach significance. This may be because it was not possible to normalise the phosphorylated receptor level to the total protein content (assay not available at the time) and as such outliers tended to skew the data.

The correlation between the activation of RTKs and downstream signalling effectors was examined (Figure 4). There was a significant correlation between the activation of C-Met with the level of P-Erk $(P=0.0005)$ and P-PI3K:T-PI3K ratio $(P=0.0037)$. There was no significant correlation between the activation status of IGFR and HER3 with downstream signalling molecules, and no significant correlation was seen between the activation status of any receptor and P-Akt. This would suggest that activation of C-Met may be a mechanism of Lapatinib resistance in vivo with an $R^{2}$ value of 0.914 for P-Erk (Figure 4Ai) and 0.84 for P-PI3K:T-PI3K (Figure 4Aii).

Whole genome sequencing from a treatment naive sample was available for one of the four patients, with persistent MAPK and PI3K pathway activation, which was related to MET activation (Figure 4Ai and Aii). There were amplifications in EGFR and HER2 (total copy number 3 for both with a single copy in the minor allele), but the
A

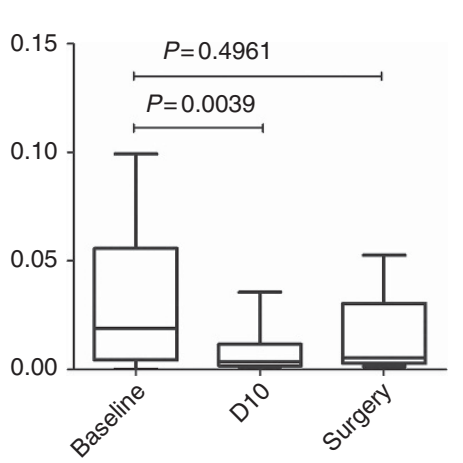

D

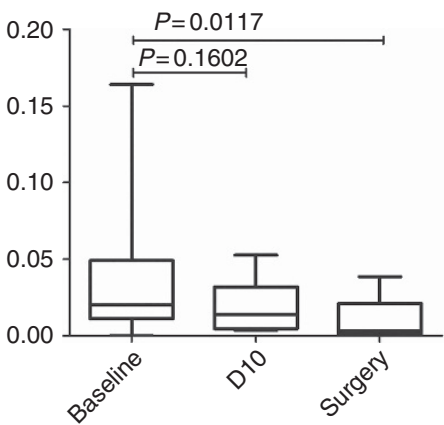

B

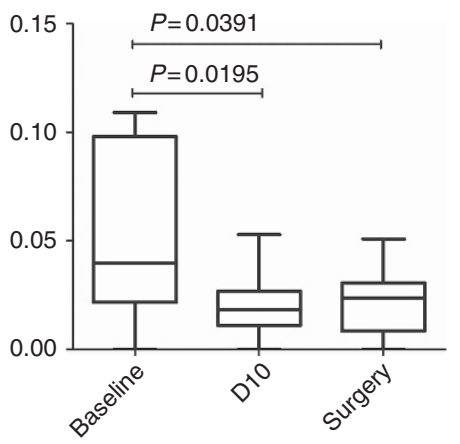

E

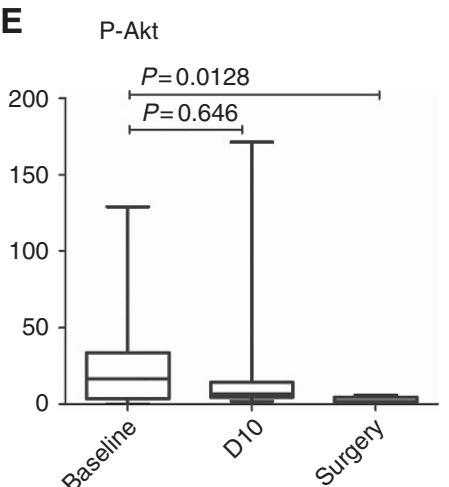

C P-p95HER2:T-p95HER2 ratio

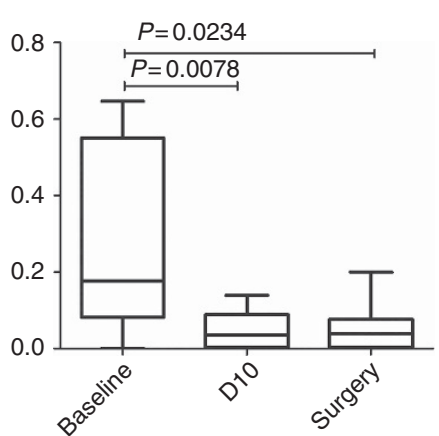

F P-Erk

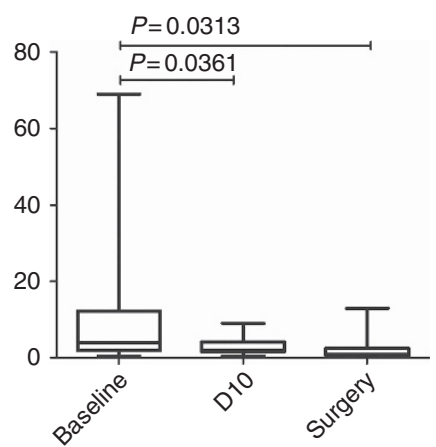

Figure 3. Phosphorylated:total (P:T) receptor ratios for HER2 (A), EGFR (B), and p95HER2 (C), along with the downstream molecules P-PI3K: T-PI3K ratio (D), P-Akt (E), and P-Erk (F) at baseline, D10 and surgery. 

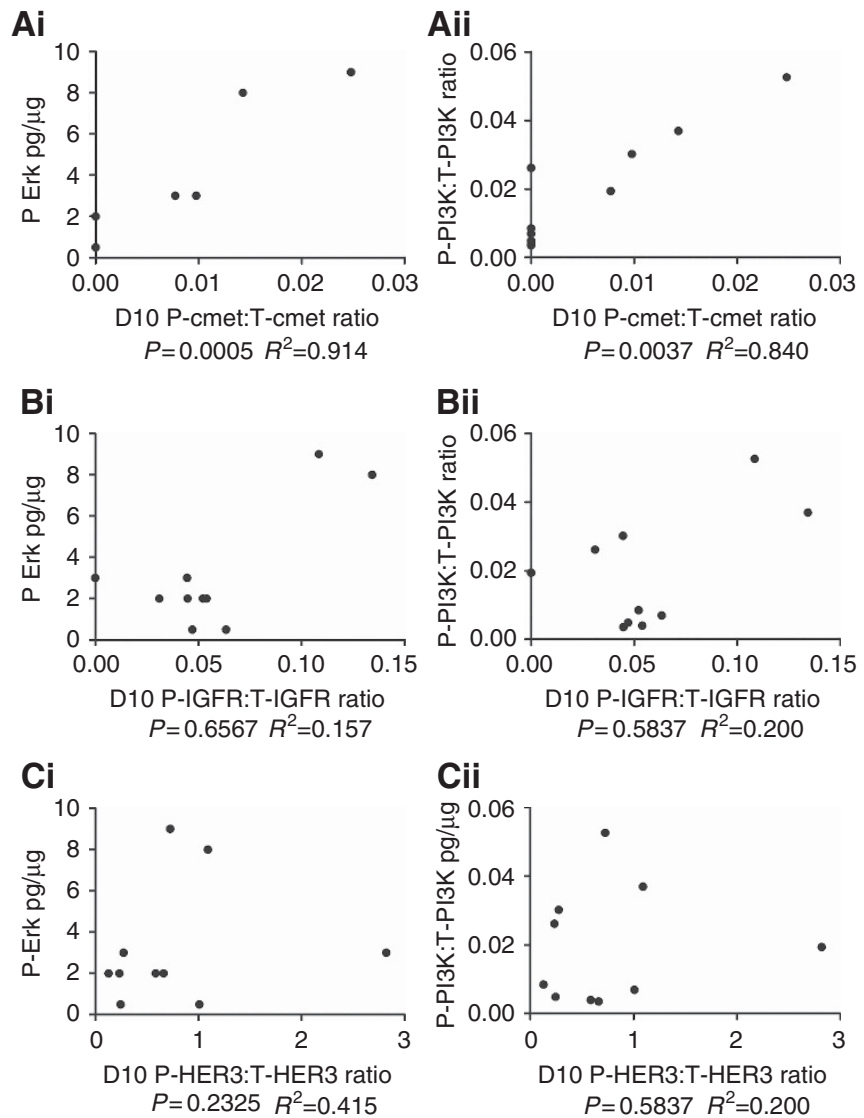

Figure 4. The ratio of phosphorylated to total protein amount representing the activation status of c-met (A), IGFR (B), and HER3 (C), plotted against the activated downstream signalling molecules P-Erk (i), and P-PI3K:T-PI3K (ii) ratio.

greatest copy number increase was seen with MET with a total copy number of 4 . KEGG output using single nucleotide variation data revealed significant clustering in the Phosphotidylinositol signalling and Wnt pathways, although no mutations were observed in the growth proliferative canonical parts of these pathways.

\section{DISCUSSION}

There are 7089 new cases of gastric cancer per year in the UK and 8332 for oesophageal cancer in the UK (http:// info.cancerresearchuk.org/cancerstats/survival/latestrates/). In the ToGa trial, which is the largest assessment of HER2 positivity in this patient group, $16.6 \%$ of tumours were HER2 positive. This would mean that 2560 patients a year in the UK would be HER2 amplified. At present, the ToGa trial has established the use of Trastuzumab in the metastatic setting (Bang et al, 2010), but there is no trial evidence to support the use of HER2 targeted therapies in patients undergoing treatment with curative intent. The use of Lapatinib has been unsuccessful in prolonging survival in metastatic patients with HER2 overexpressing OGA (Iqbal et al, 2011; Hecht et al, 2013; Satoh et al, 2014; Lorenzen et al, 2015).

The Lapatinib in early oesophago-gastric cancer trial assessed the use of Lapatinib in the peri-operative setting where median overall survival was 32.4 months. This compares with previous trials where surgery alone and in combination with peri-operative chemotherapy demonstrated median overall survival rates of 18-24 months (Cunningham et al, 2006; Allum et al, 2009). Lapatinib monotherapy was well tolerated and the main grade 3 toxicity resulted from the combination with chemotherapy. Grade 3 toxicities such as nausea/vomiting and mucositis occurred at a similar rate between the Lapatinib in early oesophago-gastric cancer patients and those seen with ECX alone in the MAGIC trial at around $20 \%$ and 10\%, respectively (Cunningham et al, 2006). Rates of grade $\geqslant 3$ diarrhoea was higher within the Lapatinib in early oesophago-gastric cancer trial which is in keeping with the fact that both Lapatinib and Capecitabine can induce diarrhoea individually, and was similar to the rates seen in the TRIO-013/ LOGiC trial (Hecht et al, 2013). The most significant toxicities were the two anastomotic leaks, which is a much higher rate than observed in the UK national audit (Chadwick et al, 2013), which was $7.4 \%$ for oesophagectomies and $4.4 \%$ for gastrectomies. Although with such small numbers in the trial it is difficult to draw conclusions, a drug effect could not be excluded for this serious complication that resulted in death of one patient, especially in view of the persistent inhibition of HER2 and EGFR at the time of surgery.

One of the study endpoints was to examine if an ex vivo assay could help predict treatment response. This was done using an organ culture, in which a whole biopsy is treated with Lapatinib for an hour, and then fixed in formalin for IHC analysis. The ex vivo assay accurately predicted in vivo P-HER2 response in $88.9 \%$ of cases, and for $100 \%$ of the cases for P-EGFR. This met the predefined study target of concordance of $75 \%$. This approach may warrant further consideration in other clinical trials.

The analysis comparing baseline predictors of sensitivity, namely IHC staining for HER2 and EGFR staining intensity, and biomarkers of resistance (C-MET staining) failed to show any correlation with clinical outcomes, namely CT response by RECIST criteria, R0 resection rates or complete pathological response. This is in keeping with the ToGa trial (Bang et al, 2010) where baseline HER2 scoring did not correlate with sensitivity to anti-HER2 directed therapy. The reasoning for this is thought to be the large amount of heterogeneity observed in staining patterns in Upper GI adenocarcinomas when compared with other cancers. Indeed, similar heterogeneity in staining was observed in this study, highlighting the need to use robust and validated scoring systems such as those used in this study (Koopman et al, 2015).

Further samples at baseline, D10 and surgery were analysed using the CEER assay, which has previously been shown to be much more sensitive for determining activation levels compared with IHC (Lee et al, 2013a). The window design of this trial also confirms that 10 days of monotherapy can give information of ontarget effects of the drug, as well as assaying potential primary resistance mechanisms. Compared with other methods of assaying drug sensitivity, such as establishment of cell lines and organoids, repeat endoscopic biopsies after a window period is relatively simple, cheap, and effective method of getting molecular response data in a timeframe that can be clinically relevant for the patient. The effect of Lapatinib was first examined on its primary targets, namely HER2 and EGFR. After 10 days of Lapatinib monotherapy, there was a significant reduction in the proportion of activated receptors for both HER2 and EGFR. This is particularly important as it appears to precede any metabolic response as no response was observed by PET imaging over this same time period. There was also significant inhibition of $\mathrm{p} 95$, the truncated form of HER2 which lacks the extracellular domain. In breast cancer, resistance to Trastuzumab has been partly attributed to p95 (Duchnowska et al, 2014), as the antibody is unable to bind the protein due to the lack of an extracellular domain, but there was significant inhibition of p95 noted in this study by Lapatinib at day 10, which continued through to surgery. This highlights that repeat biopsy after a window period of monotherapy can provide the earliest evidence of on-target action for Lapatinib, and the drug also significantly inhibits p95.

A 4-6 week wash out period before surgery was used to ensure that the patients had recovered fully from the neoadjuvant therapy 
before definitive surgery. This period has typically been used with cytotoxic chemotherapy as this usually allows for complete marrow recovery. Similar pre-operative wash out periods have also been used with anti-HER2 agents such as Trastuzumab in breast cancer, and have not been associated with an increased operative morbidity (Gianni et al, 2010). The CEER data demonstrated that despite a wash out period of several weeks, there was still significant reduction in EGFR signalling at the time of surgery, with a further nonsignificant reduction in HER2 signalling. Although the small numbers make it impossible to draw any definitive conclusion, it is possible that this persistent inhibition of growth factor signalling pathways at surgery may have contributed to the anastomotic leak rate. It should therefore be considered whether the wash out period required for neoadjuvant therapy using Lapatinib needs to be longer than typically used for traditional chemotherapeutics. This is particularly noteworthy given that there are current trials open to recruitment using Lapatinib in a similar context with a similar wash out period (Cunningham, 2012).

Not only is there evidence of receptor deactivation in response to drug but a decrease in the activation of downstream molecules such as PI3K, P-Erk, and P-Akt. This supports the on-target action of Lapatinib on these tumours reducing EGFR and HER2 mediated signalling by PI3K and with a trend for reduction of the MAPK pathway.

Preclinical studies have pointed to RTK crosstalk from MET as being an important mechanism of resistance to Lapatinib and other HER2 directed therapies (Shattuck et al, 2008; Chen et al, 2012; Lee et al, 2013b; Paterson et al, 2013b; Liu et al, 2014; Ha et al, 2014; Zhang et al, 2014). This study provides some of the first in vivo evidence that, $\mathrm{MET}$ appears to be the most important RTK in activating proliferative pathways (PI3K and MAPK) in the presence of Lapatinib inhibition of EGFR and HER2. This was demonstrated by the CEER assay where the activation of the MAPK and PI3K pathways after 10 days of Lapatinib monotherapy was significantly correlated with the activation of MET, but not with IGFR or HER3 (Figure 4). Larger studies would need to be undertaken to fully understand whether this molecular crosstalk has implications on clinical outcome as this trial was not powered to detect this. Our cohort is also not large enough to adequately correct for differences in staging, or to perform a multiple regression analysis to correlate clinical outcomes and molecular findings.

Whole genome sequencing was available for one of the patients with persistent mitogenic signalling in the presence of Lapatinib, which appeared to be due to MET activation. The main canonical pathways for PI3K and MAPK were intact in keeping with previous genomic studies in OGA have suggested that point mutations do not tend to drive proliferation (Dulak et al, 2013; Weaver et al, 2014). Copy number data from this same patientdemonstrated HER2 and EGFR amplification (total copy number of 3 for each), but interestingly, the most amplified of the RTKs was MET. This supports the finding that activation of these mitogenic pathways at D10 is more likely to be due to RTK crosstalk from MET by amplification.

These in vivo findings suggests that MET mediates Lapatinib resistance and is particularly noteworthy given recent interest in using MET inhibitors. These trials have failed to demonstrate efficacy of single agent MET TKIs (Shah et al, 2013) and MET targeting antibodies (Cunningham et al, 2015), so it is likely that combination therapies should be considered with anti-HER2 therapies used in combination with anti-MET agents in the context of well designed stratified trials (Ha et al, 2014).

This study demonstrated that 10 days of monotherapy followed by CEER analysis can reveal in vivo evidence of target activity in a simple, rapid, and cheap manner, and in addition may provide clinically useful information about patients who may be resistant, providing the information for clinicians to make management decisions in real time, and to select appropriate targeted therapy in a truly personalised manner.

\section{CONFLICT OF INTEREST}

The authors declare no conflict of interest.

\section{REFERENCES}

Allum WH, Stenning SP, Bancewicz J, Clark PI, Langley RE (2009) Long-term results of a randomized trial of surgery with or without preoperative chemotherapy in esophageal cancer. J Clin Oncol 27: 5062-5067.

Bang YJ, Van Cutsem E, Feyereislova A, Chung HC, Shen L, Sawaki A, Lordick F, Ohtsu A, Omuro Y, Satoh T, Aprile G, Kulikov E, Hill J, Lehle M, Ruschoff J, Kang YK (2010) Trastuzumab in combination with chemotherapy versus chemotherapy alone for treatment of HER2-positive advanced gastric or gastro-oesophageal junction cancer (ToGA): a phase 3, open-label, randomised controlled trial. Lancet 376: 687-697.

CancerResearchUK (2015) Oesophageal cancer incidence statistics Cancer Research UK. Available at: http://www.cancerresearchuk.org/cancer-info/ cancerstats/types/oesophagus/incidence/(accessed on 18 May 2015).

Chadwick G, Groene O, Cromwell D, Hardwich R, Riley S, Crosby T, Greenaway K (2013) National Oesophago-Gastric Audit 2013. In: England, T. R. C. O. S. O. (ed.)London.

Chen CT, Kim H, Liska D, Gao S, Christensen JG, Weiser MR (2012) MET activation mediates resistance to lapatinib inhibition of HER2-amplified gastric cancer cells. Mol Cancer Ther 11: 660-669.

CRUK. Cancer survival rates for patients diagnosed 1996e1999. Survival statistics for the most common cancers. Available at: http://info.cancerresearchuk.org/cancerstats/survival/latestrates/ (accessed 5 February 2009).

Cunningham D (2012) A Randomised Phase II/III trial of Perioperative Chemotherapy with or without Bevacizumab in Operable Oesophagogastric Adenocarcinoma. A Feasibility Study Evaluating Lapatinib in HER-2 Positive Oesophagogastric Adenocarcinomas. Protocol Version 6, 28 September 2012.

Cunningham D, Allum WH, Stenning SP, Thompson JN, Van De Velde CJ, Nicolson M, Scarffe JH, Lofts FJ, Falk SJ, Iveson TJ, Smith DB, Langley RE, Verma M, Weeden S, Chua YJ, Participants MT (2006) Perioperative chemotherapy versus surgery alone for resectable gastroesophageal cancer. $N$ Engl J Med 355: 11-20.

Cunningham D, Starling N, Rao S, Iveson T, Nicolson M, Coxon F, Middleton G, Daniel F, Oates J, Norman AR (2008) Capecitabine and oxaliplatin for advanced esophagogastric cancer. $N$ Engl J Med 358: 36-46.

Cunningham D, Tebbutt NC, Davidenko I, Murad AM, Al-Batran S-E, Ilson DH, Tjulandin S, Gotovkin E, Karaszewska B, Bondarenko I, Tejani MA, Udrea AA, Tehfe MA, Baker N, Oliner KS, Zhang Y, Hoang T, Sidhu R, Catenacci DVT (2015) Phase III, randomized, double-blind, multicenter, placebo (P)-controlled trial of rilotumumab (R) plus epirubicin, cisplatin and capecitabine (ECX) as first-line therapy in patients (pts) with advanced MET-positive (pos) gastric or gastroesophageal junction (G/GEJ) cancer: RILOMET-1 study. ASCO Meet Abstr 33: 4000.

Dobbin ZC, Katre AA, Steg AD, Erickson BK, Shah MM, Alvarez RD, Conner MG, Schneider D, Chen D, Landen CN (2014) Using heterogeneity of the patientderived xenograft model to identify the chemoresistant population in ovarian cancer. Oncotarget 5: 8750-8764.

Duchnowska R, Sperinde J, Chenna A, Haddad M, Paquet A, Lie Y, Weidler JM, Huang W, Winslow J, Jankowski T, Czartoryska-Arlukowicz B, Wysocki PJ, Foszczynska-Kloda M, Radecka B, Litwiniuk MM, Zok J, Wisniewski M, Zuziak D, Biernat W, Jassem J (2014) Quantitative measurements of tumoral P95HER2 protein expression in metastatic breast cancer patients treated with trastuzumab: independent validation of the p95HER2 clinical cutoff. Clin Cancer Res 20: 2805-2813.

Dulak AM, Stojanov P, Peng S, Lawrence MS, Fox C, Stewart C, Bandla S, Imamura Y, Schumacher SE, Shefler E, Mckenna A, Carter SL, Cibulskis K, Sivachenko A, Saksena G, Voet D, Ramos AH, Auclair D, Thompson K, Sougnez C, Onofrio RC, Guiducci C, Beroukhim R, Zhou Z, Lin L, Lin J, Reddy R, Chang A, Landrenau R, Pennathur A, Ogino S, Luketich JD, Golub TR, Gabriel SB, Lander ES, Beer DG, Godfrey TE, Getz G, Bass AJ (2013) Exome and whole-genome sequencing of esophageal 
adenocarcinoma identifies recurrent driver events and mutational complexity. Nat Genet 45: 478-486.

Fassan M, Mastracci L, Grillo F, Zagonel V, Bruno S, Battaglia G, Pitto F, Nitti D, Celiento T, Zaninotto G, Fiocca R, Rugge M (2012) Early HER2 dysregulation in gastric and oesophageal carcinogenesis. Histopathology 61: 769-776.

Fischer A, Vazquez-Garcia I, Illingworth CJ, Mustonen V (2014) Highdefinition reconstruction of clonal composition in cancer. Cell Rep 7: $1740-1752$.

Gianni L, Eiermann W, Semiglazov V, Manikhas A, Lluch A, Tjulandin S, Zambetti M, Vazquez F, Byakhow M, Lichinitser M, Climent MA, Ciruelos E, Ojeda B, Mansutti M, Bozhok A, Baronio R, Feyereislova A, Barton C, Valagussa P, Baselga J (2010) Neoadjuvant chemotherapy with trastuzumab followed by adjuvant trastuzumab versus neoadjuvant chemotherapy alone, in patients with HER2-positive locally advanced breast cancer (the NOAH trial): a randomised controlled superiority trial with a parallel HER2-negative cohort. Lancet 375: 377-384.

Ha SY, Lee J, Jang J, Hong JY, Do IG, Park SH, Park JO, Choi MG, Sohn TS, Bae JM, Kim S, Kim M, Park CK, Kang WK, Kim KM (2014) HER2positive gastric cancer with concomitant MET and/or EGFR overexpression: A distinct subset of patients for dual inhibition therapy. Int J Cancer 136: 1629-1635.

Hecht JR, Bang YJ, Qin S, Chung HC, Xu JM, Park JO, Jeziorski K, Shparyk Y, Hoff PM, Sobrero AF, Salman P, Li J, Protsenko S, Buyse ME, Afenjar K, Kaneko T, Kemner A, Santillana S, Press MF, Slamon DJ (2013) Lapatinib in combination with capecitabine plus oxaliplatin (CapeOx) in HER2positive advanced or metastatic gastric, esophageal, or gastroesophageal adenocarcinoma (AC): The TRIO-013/LOGiC Trial. J Clin Oncol 31: Abstract LBA4001.

Iqbal S, Goldman B, Fenoglio-Preiser CM, Lenz HJ, Zhang W, Danenberg KD, Shibata SI, Blanke CD (2011) Southwest Oncology Group study S0413: a phase II trial of lapatinib (GW572016) as first-line therapy in patients with advanced or metastatic gastric cancer. Ann Oncol 22: 2610-2615.

Koopman T, Louwen M, Hage M, Smits MM, Imholz AL (2015) Pathologic diagnostics of HER2 positivity in gastroesophageal adenocarcinoma. Am J Clin Pathol 143: 257-264.

Larson DE, Harris CC, Chen K, Koboldt DC, Abbott TE, Dooling DJ, Ley TJ, Mardis ER, Wilson RK, Ding L (2012) SomaticSniper: identification of somatic point mutations in whole genome sequencing data. Bioinformatics 28(3): 311-317.

Lee J, Kim S, Kim P, Liu X, Lee T, Kim KM, Do IG, Park JO, Park SH, Jang J, Hoe N, Harvie G, Kuller A, Jain A, Meyer G, Leesman G, Park YS, Choi MG, Sohn TS, Bae JM, Lim HY, Singh S, Kang WK (2013a) A novel proteomicsbased clinical diagnostics technology identifies heterogeneity in activated signaling pathways in gastric cancers. PLoS One 8: e54644.

Lee YY, Kim HP, Kang MJ, Cho BK, Han SW, Kim TY, Yi EC (2013b) Phosphoproteomic analysis identifies activated MET-axis PI3K/AKT and MAPK/ERK in lapatinib-resistant cancer cell line. Exp Mol Med 45: e64.

Li D, Wen X, Ghali L, Al-Shalabi FM, Docherty SM, Purkis P, Iles RK (2008) hCG beta expression by cervical squamous carcinoma-in vivo histological association with tumour invasion and apoptosis. Histopathology 53: $147-155$.

Liu YJ, Shen D, Yin X, Gavine P, Zhang T, Su X, Zhan P, Xu Y, Lv J, Qian J, Liu C, Sun Y, Qian Z, Zhang J, Gu Y, Ni X (2014) HER2, MET and FGFR2 oncogenic driver alterations define distinct molecular segments for targeted therapies in gastric carcinoma. Br J Cancer 110: 1169-1178.

Lordick F, Ott K, Krause BJ, Weber WA, Becker K, Stein HJ, Lorenzen S, Schuster T, Wieder H, Herrmann K, Bredenkamp R, Hofler H, Fink U, Peschel C, Schwaiger M, Siewert JR (2007) PET to assess early metabolic response and to guide treatment of adenocarcinoma of the oesophagogastric junction: the MUNICON phase II trial. Lancet Oncol 8: 797-805.

Lorenzen S, Riera Knorrenschild J, Haag GM, Pohl M, Thuss-Patience P, Bassermann F, Helbig U, Weissinger F, Schnoy E, Becker K, Stocker G, Ruschoff J, Eisenmenger A, Karapanagiotou-Schenkel I, Lordick F (2015) Lapatinib versus lapatinib plus capecitabine as second-line treatment in human epidermal growth factor receptor 2 -amplified metastatic gastro- oesophageal cancer: a randomised phase II trial of the Arbeitsgemeinschaft Internistische Onkologie. Eur J Cancer 51: 569-576.

Nik-Zainal S, Van Loo P, Wedge DC, Alexandrov LB, Greenman CD, Lau KW, Raine K, Jones D, Marshall J, Ramakrishna M, Shlien A, Cooke SL, Hinton J, Menzies A, Stebbings LA, Leroy C, Jia M, Rance R, Mudie LJ, Gamble SJ, Stephens PJ, Mclaren S, Tarpey PS, Papaemmanuil E, Davies HR, Varela I, Mcbride DJ, Bignell GR, Leung K, Butler AP, Teague JW, Martin S, Jonsson G, Mariani O, Boyault S, Miron P, Fatima A, Langerod A, Aparicio SA, Tutt A, Sieuwerts AM, Borg A, Thomas G, Salomon AV, Richardson AL, Borresen-Dale AL, Futreal PA, Stratton MR, Campbell PJ (2012) The life history of 21 breast cancers. Cell 149: 994-1007.

Paterson AL, O'Donovan M, Provenzano E, Murray LJ, Coleman HG, Johnson BT, Mcmanus DT, Novelli M, Lovat LB, Fitzgerald RC (2013a) Characterization of the timing and prevalence of receptor tyrosine kinase expression changes in oesophageal carcinogenesis. J Pathol 230: 118-128.

Paterson AL, Shannon NB, Lao-Sirieix P, Ong CA, Peters CJ, O'Donovan M, Fitzgerald RC (2013b) A systematic approach to therapeutic target selection in oesophago-gastric cancer. Gut 62: 1415-1424.

Sachs N, Clevers H (2014) Organoid cultures for the analysis of cancer phenotypes. Curr Opin Genet Dev 24: 68-73.

Satoh T, Xu RH, Chung HC, Sun GP, Doi T, Xu JM, Tsuji A, Omuro Y, Li J, Wang JW, Miwa H, Qin SK, Chung IJ, Yeh KH, Feng JF, Mukaiyama A, Kobayashi M, Ohtsu A, Bang YJ (2014) Lapatinib plus paclitaxel versus paclitaxel alone in the second-line treatment of HER2-amplified advanced gastric cancer in Asian populations: TyTAN-a randomized, phase III study. J Clin Oncol 32: 2039-2049.

Shah MA, Wainberg ZA, Catenacci DV, Hochster HS, Ford J, Kunz P, Lee FC, Kallender H, Cecchi F, Rabe DC, Keer H, Martin AM, Liu Y, Gagnon R, Bonate P, Liu L, Gilmer T, Bottaro DP (2013) Phase II study evaluating 2 dosing schedules of oral foretinib (GSK1363089), cMET/VEGFR2 inhibitor, in patients with metastatic gastric cancer. PLoS One 8: e54014.

Shattuck DL, Miller JK, Carraway 3rd KL, Sweeney C (2008) Met receptor contributes to trastuzumab resistance of Her2-overexpressing breast cancer cells. Cancer Res 68: 1471-1477.

Van Cutsem E, Bang YJ, Feng-Yi F, Xu JM, Lee KW, Jiao SC, Chong JL, Lopez-Sanchez RI, Price T, Gladkov O, Stoss O, Hill J, Ng V, Lehle M, Thomas M, Kiermaier A, Ruschoff J (2014) HER2 screening data from ToGA: targeting HER2 in gastric and gastroesophageal junction cancer. Gastric Cancer 18: 476-484.

Van Loo P, Nordgard SH, Lingjaerde OC, Russnes HG, Rye IH, Sun W, Weigman VJ, Marynen P, Zetterberg A, Naume B, Perou CM, Borresen-Dale AL, Kristensen VN (2010) Allele-specific copy number analysis of tumors. Proc Natl Acad Sci USA 107: 16910-16915.

Wainberg ZA, Anghel A, Desai AJ, Ayala R, Luo T, Safran B, Fejzo MS, Hecht JR, Slamon DJ, Finn RS (2010) Lapatinib, a dual EGFR and HER2 kinase inhibitor, selectively inhibits HER2-amplified human gastric cancer cells and is synergistic with trastuzumab in vitro and in vivo. Clin Cancer Res 16: 15091519.

Weaver JMJ, Ross-Innes CS, Shannon N, Lynch AG, Forshew T, Barbera M, Murtaza M, Ong C-AJ, Lao-Sirieix P, Dunning MJ, Smith L, Smith ML, Anderson CL, Carvalho B, O'Donovan M, Underwood TJ, May AP, Grehan N, Hardwick R, Davies J, Oloumi A, Aparicio S, Caldas C, Eldridge MD, Edwards PAW, Rosenfeld N, Tavare S, Fitzgerald RC, The OC (2014) Ordering of mutations in preinvasive disease stages of esophageal carcinogenesis. Nat Genet 46: 837-843.

Zhang Z, Wang J, Ji D, Wang C, Liu R, Wu Z, Liu L, Zhu D, Chang J, Geng R, Xiong L, Fang Q, Li J (2014) Functional genetic approach identifies MET, HER3, IGF1R, INSR pathways as determinants of lapatinib unresponsiveness in HER2-positive gastric cancer. Clin Cancer Res 20: 4559-4573.

This work is published under the standard license to publish agreement. After 12 months the work will become freely available and the license terms will switch to a Creative Commons AttributionNonCommercial-Share Alike 4.0 Unported License. 